\title{
Preparation and Properties of Polyester Based Nanocomposites with Good Air-tightness
}

\author{
Fusheng Luo \\ Department of Chemical Engineering, Huizhou University \\ Guangdong 516007, China
}

Tel: 86-752-252-7229Ｅ-mail: lfs@hzu.edu.cn

\begin{abstract}
The research is financed by China National key fundamental Research Foundation (2004CB217808) and Scientific Research Foundation of Huizhou University (No505.0302).
\end{abstract}

\begin{abstract}
The permeability of polymer is determined by its chain configuration, phase morphology and the interaction with the penetrant. Based on these principles, the permeability of the polyester of poly (ethylene terephthalate)(PET) is enhanced through different modifiers in order to apply it to barrier packing bottle. In this paper, poly (m-xylylene adipamide-terephthalyl amide) (MXD6T) and nanometer silicon dioxide $\left(\mathrm{SiO}_{2}\right)$ nanocomposite with $\mathrm{SiO}_{2}$ load from 5\% to $20 \%$ (by mass) is prepared and used to improve the barrier property of polyester bottle materials. In preparing MXD6T-SiO 2 (NMXD6T), silica is in-situ polymerized with hexane diacid, terephthalic acid (TPA), alkyl and aromatic diamine. The different molar ratio of hexane diacid to terephthalic acid with the same molar diamine is designed to give the composites with different viscosity, thermal performance and the barrier properties. The 3-8 times of enhanced permeability of these polyester matrix nanocomposites with this NMXD6T to pure polyester resins are reported.

The relationship of NMXD6T's configuration and the permeability properties of polyester nanocomposites are characterized by techniques of the dynamic scanning calorimeter (DSC), FT Infrared, and Transmission Electronic Microscopy (TEM). DSC shows that NMXD6T is totally amorphous. FTIR shows the existence of silicon-oxygen bond formed in the nanocomposite, and TEM indicates an average size from $30 \mathrm{~nm}$ to $100 \mathrm{~nm}$ of $\mathrm{SiO}_{2}$ particles being phase separated from MXD6T matrix, while well bonded to the polyester chains. It is concluded that the NMXD6T-PET nanocomposites may be one of the best way to enhance the barrier properties of polyesters for their packing application.
\end{abstract}

Keywords: Nylon MXD6T, Silicon dioxide, PET, Nanocomposites

\section{Introduction}

In packing industry, the research of high barrier properties polymer materials is of increasing interest to prevent gases like oxygen, carbon dioxide or water vapor from permeating through the materials. In generally, the permeability of polymer is determined by penetrant, polymer's configuration and properties, the interaction of penetrant and polymer (Ding Yun-sheng, 2001, p.360-368. Soney C Georgy, 2000, p. 2136-2145 \& Hongchul Seo, 2000, p. 2714-2720). Material's microcracks, pinholes and defects can increase the penetrability of micromolecules. Oxygen permeation, which is of great importance for packing purpose, is almost entirely defect-dominated (A. Gruniger, 2004, p. 308-315. A.P. Roberts, 2002, p. 75-82). Also, the free volume of polymer has important influence on the permeation of micromolecules through polymer matrix: the larger free volume is, the stronger penetrability is. Other important factors are polymer's surface polarity, chain rigidity and cohesion (He Zuoyun, 1996, p. 17-23). Temperature and humidity are exterior effect factors. For most polymers, the contributions of microcracks, pinholes and defects to permeation increase with increasing temperature and humidity. However, the penetrability of amorphous nylon decreases with increasing humidity. U. S. Pat. NO. 637, 659,1 describes the combination of at least two of the diacids with the diamine effectively disrupts the crystallization tendency of these macromolecules to allow clear transparent amorphous structures to be maintained throughout the processing steps (Lan, Tie, 1999).

Poly (m-xylylene adipamide) (MXD6), a semicrystalline polymer, is prepared by m-xylylenediamine and hexane diacid. It is one of the high barrier property materials with good properties: high gas barrier (even in the high humidity surrounding, it also has outstanding barrier property (CHEN Yan, 1997, p. 73-78)); high heat stability; good preserving verdure property; proper crystallization speed; and good moulding machining property, which make it possible to be applied to several fields in packing industry (especially for multilayer containers), automobiles and magnetism materials. 
Several reports showed that the oxygen penetrate rate of MXD6 biaxially oriented film is one tenth to one twentieth of that of nylon 6 biaxially oriented film and one twentieth to one twenty fifth of that of PET biaxially oriented film (WANG Yunqiu, 1997, p. 43-49). Also, for carbon dioxide, the penetrate rate of MXD6 is one fifth of that of PET. Moreover, when the amount of MXD6 is about 10\% weight in PET, the barrier properties of PET can be improved nearly two times.

Nanocomposites technology has been described as the next great frontier of material science. The principle that uses a nanometer material to enhance or improve the properties of a polymer matrix is researched by many people. Employing minimal addition levels $(<10 \mathrm{wt} \%)$ nanoclays enhance mechanical, thermal, dimensional and barrier performance properties significantly (Maxfield, MacRae, 1992). The use of dispersed clay in matrix such as MXD6 of the inner layer a multilayer container may reduce the haze of multilayer structures and improve the appearance of oriented films and molded articles, including bottles. But clay particles in a nylon-6 nanocomposite induce crystallization and lead to void and holes formation in the polymer films, which decrease the barrier properties of these composites (FANG Sheng xing, 2001, p. 24-28). For MXD6 has higher dispersity in PET, terephthalic acid was added to get poly (m-xylylene adipamide-terephthalyl amide) (MXD6T).

In this paper, MXD6T- $\mathrm{SiO}_{2}$ (NMXD6T) nanocomposites with $\mathrm{SiO}_{2}$ load from $5 \%$ to $20 \%$ (wt) is prepared, and the influences of its properties and morphology on barrier properties are investigated. The structure and properties of NMXD6T are investigated by TEM, DSC and FITR, etc. The NMXD6T was added in PET to prepare NMXD6T-PET(NPET). The measurement of he air permeability of PET and NPET showed that the barrier properties of NPET were enhanced obviously 3-8 times.

\section{Materials and methods}

\subsection{Materials}

Monomer of m-xylylenediamine (analytical reagent) was purchased from Mitsubishi Gas Chemical (MGC), while hexane diacid (chemical reagent) from Beijing Xingjin Chemical Company, terephthalic acid (TPA) (Tianjin-Yiheng Technology Co. Ltd). Ethyl silicate hydrolyzed to prepare nanometer $\mathrm{SiO}_{2}$ particles was chemical reagent from Beijing Yili Fine Chemical Co. Ltd.

\subsection{Preparation of MXD6T-SiO ${ }_{2}$ and NPET nanocomposites}

The MXD6T-SiO 2 nanocomposite was prepared by a two-step method. First, ethyl silicate was hydrolyzed to prepare $\mathrm{SiO}_{2}$ particles, in which liquid ethyl silicate was added dropwise to a solution mixture of m-xylylenediamine, hexane diacid, terephthalic acid and distilled water. The molar ratio of acid to diamine was maintained at 1.0. The liquid-solid mixture was stirred for at least $30 \mathrm{~min}$ at room temperature to form a homogenate system. Then, the obtained homogenate was heated up to $98^{\circ} \mathrm{C}$ gradually under the protection of nitrogen gas and started dewatering about $1 \mathrm{~h}$ until this liquid system became a solid one. After that, dewatering process was kept on and temperature was increased from $100^{\circ} \mathrm{C}$ to $220^{\circ} \mathrm{C}$ for $1-1.5 \mathrm{~h}$. At last, the second step polymerization reaction began and lasted for $1.5-2 \mathrm{~h}$ under $240^{\circ} \mathrm{C}$ to $260^{\circ} \mathrm{C}$. The final product was obtained at the protection of nitrogen gas.

The MXD6T- $\mathrm{SiO}_{2}$ nanocomposites (wt-10\%) were sufficiently mixed with PET powder by ball mill to obtain the new composite materials (NPET). NPET were pressed into film with $0.1 \mathrm{~mm}$ thickness by tablet at $275^{\circ} \mathrm{C}$.

\subsection{Preparation of samples}

NMXD6T was smashed in disintegrator and vacuum-dried at $60^{\circ} \mathrm{C}$ for $4 \mathrm{~h}$. Then NMXD6T small particles were sealed in self- given bags and saved in desiccators.

\section{Characterization}

DSC: DSC patterns were measured by a differential scanning calorimeter (NETZSCH STA 409 PC) from room temperature to $360^{\circ} \mathrm{C}$ at a heating rate of $10^{\circ} \mathrm{C} /$ min with error of $\pm 1^{\circ} \mathrm{C}$.

FITR: The FTIR spectra of the MXD6T and NMXD6T were determined by a MAGNA-AR560E.S.P FTIR spectrometer. TEM: TEM observation operated under Hitachi 800.

Intrinsic Viscosity: MXD6T and NMXD6T samples were dissolved in mixed solvents of 50/50 (by mass) of 1, 1, 2, 2-tetracholoro ethane and phenol, and then measured with U II -man viscometer with a concentration of $0.001 \mathrm{~g} \cdot \mathrm{ml}^{-1}$ at temperature of $(25 \pm 0.1)^{\circ} \mathrm{C}$.

Gas permeation properties: Gas permeation Tester, Jinan blue light Electrical and Mechanical Technology Co., Ltd.

\section{Results and discussion}

\subsection{The influences of acid ratio and $\mathrm{SiO}_{2}$ content on nanocomposites viscosity}

The influences of TPA content and $\mathrm{SiO}_{2}$ content on polymer viscosity are shown in Fig.1. In Fig.1A, it can be found that the viscosity of nanocomposites decreases with decreasing TPA. It is well known that increase of aliphatic acid can 
improve the flexibility of the polymer. While the increase of the aromatic acid can increase the rigidity of the polymer chain which also means the improvement of polymer barrier properties. Hence, perfect acid ratio and $\mathrm{SiO}_{2}$ content are important for betterment of barrier property and polymerization degree. So, to balance barrier property and polymerization degree, the content of TPA is from $20 \%$ to $50 \%$ (by mole) and $\mathrm{SiO} 2$ content is from $5 \%$ to $20 \%$ (by mass).

\subsection{Bonds of MXD6T and NMXD6T}

The FITR spectrum figures of MXD6T and NMXD6T are shown in Fig2. The appearance of the absorption bands at $1643 \mathrm{~cm}^{-1}\left(\mathrm{C}=\mathrm{O}\right.$ bond stretch of amido group), $1546 \mathrm{~cm}^{-1}$ (C-N bond stretch of amido group) (part A) and $1643 \mathrm{~cm}^{-1}$, $1547 \mathrm{~cm}^{-1}$ (part B) confirmed the formation of amido group. The amido group is strong polarity bond so it has good barrier properties to nonpolarity gas, such as oxygen gas and carbon dioxide. Comparing (Fig.1A) with (Fig.1B), 1030 $\mathrm{cm}^{-1}$ and $868 \mathrm{~cm}^{-1}$ (Si-O bond absorption) confirm the formation of composite system which has strong interaction between inorganic phase $\mathrm{SiO}_{2}$ and organic phase polymer matrix.

\subsection{Glass transition temperature of oligomers}

The increase of glass transition temperature $\left(\mathrm{T}_{\mathrm{g}}\right)$ reflects the rigidity increase of polymer chain; meanwhile, the increase of rigidity enhances the polymer barrier properties. The glass transition temperatures of the oligomers can be obtained form the DSC curves showed in Fig.3. In Fig.3A, it can be found that the $\mathrm{T}_{\mathrm{g}}$ values shift to higher temperatures with the increase of $\mathrm{SiO}_{2}$ content. While in Fig.3B, the $\mathrm{T}_{\mathrm{g}}$ values decrease with the increasing ratio of hexane diacid to TPA. The dependence of $\mathrm{T}_{\mathrm{g}}$ values on $\mathrm{SiO}_{2}$ and TPA indicated that the incorporation of nanoparticles and TPA into polymer matrix have direct effects on its chain rigidity.

\subsection{Dispersion of $\mathrm{SiO}_{2}$ nanoparticles in polymer matrix}

The interspace of polymer matrix macromolecules can be filled by nanometer particles dispersed homogeneously. Figure 4 shows the TEM morphology of NPET. In figure 4A, it can be found that most of particles are dispersed homogeneously. But there are black agglomerations in some areas.Fig4B is the enlargement of these agglomerations. The enlarged TEM graph showed that the particles also exist in nanometer dimension.

\subsection{The air permeability of the polyester}

Table 1 shows the gas permeability of PET and NPET. It can be found that the permeability can be enhanced by the addition of nanometer particles $\mathrm{SiO}_{2}$ and the increase of TPA. The results indicated that the NMXD6T-PET nanocomposites can be a potential material in packing application for their enhanced air-tightness property.

\section{Conclusions}

In conclusion, a new nanocomposites system with different TPA content and $\mathrm{SiO}_{2}$ content were prepared and used as a modifier to improve barrier property of PET. The measurement of the viscosity and $\mathrm{T}_{\mathrm{g}}$ values showed the influence of TPA and $\mathrm{SiO}_{2}$ content. The investigation of TEM indicated that $\mathrm{SiO}_{2}$ particles exist in nanometer dimension in the polymer matrix. The enhanced the air-tightness properties of polyesters revealed that the NMXD6T-PET nanocomposites can be a potential material in packing application.

\section{References}

Ding, Yunsheng, Zhang, Zhicheng, Shi, Tiejun. Progress and Research of Impermeable Polymer Materials. (2001). Jounral of Functional Polymers. 14, 360-368.

Soney C Georgy, G Groeninckx, K N Ninan, et al.. Molecular transport of aromatic hydrocarbons through nylon- 6/ ethylene propylene rubber blends. (2000). J Polym S CI: Part B: Polym Physics. 38, 2136-2145.

Hongchul Seo, Jongho Jeon, Yong Gun Shul, et al. Water sorption and activation energy in polyimide thin films. (2000). J Polym S CI: Part B: Polym Physics. 38, 2714-2720.

A. Gruniger, Ph. Rudolf von Rohr. Influence of defects in $\mathrm{SiO}_{2}$ thin films on their barrier properties. (2004). Thin Solid Films. 308-315.

A.P. Roberts, B.M. Henry, A.P. Sutton, et al.. The minute development direction of high barrier properties resins and materials. (2002). J. Membr Sci. 208, 75-82.

He, Zuoyun, Xion Yuanfan, Yang Yuehui. The development of preparation of barrier nylon resins. (1996). China Plastics. 10. 17-23.

Lan, Tie, Psihogios. (1999). US6376591.

Chen, Yan, Wan, Xinyu, et al. Study on polyimide/ silica nanocomposite films. (1997). Acta polymeica sinica. 11, 73-78. 
Wang, Yunqiu. A new ultra-high functional nylon MXD6 resin. (1997). Modern plastics processing and applications. 9 , 43-49.

Maxfield, MacRae, Christiani, et.al.. 1992. US5385776.

Fang, Shengxing \& Hu, Yingmei. Manufacturing Scheme of Plastic Beer Bottles. (2001). Plastics. 30, 24-28.

Table 1. The comparison of gas permeation properties for PET and NPET

\begin{tabular}{ccccc}
\hline Sample & $\mathrm{SiO}_{2}$ & air permeability & $\mathrm{O}_{2}$ & $\mathrm{CO}_{2}$ \\
$\mathrm{~T}\left(25^{\circ} \mathrm{C}\right)$ & $(\%)$ & $/\left[\mathrm{cm}^{3} \cdot \mathrm{m}^{-2} \cdot(24 \mathrm{~h} \cdot \mathrm{atm})^{-1}\right]$ & & \\
\hline PET & & & 153 & 576 \\
SNPET -1 & $\mathrm{SiO}_{2}(10 \%)$ & $\mathrm{A} / \mathrm{TPA}=3$ & 62 & 195 \\
SNPET -2 & $\mathrm{SiO}_{2}(10 \%)$ & $\mathrm{A} / \mathrm{TPA}=2$ & 45 & 154 \\
SNPET -3 & $\mathrm{SiO}_{2}(10 \%)$ & $\mathrm{A} / \mathrm{TPA}=1$ & 37 & 112 \\
SNPET -4 & $\mathrm{SiO}_{2}(20 \%)$ & A/TPA $=1$ & 19 & 72 \\
\hline
\end{tabular}

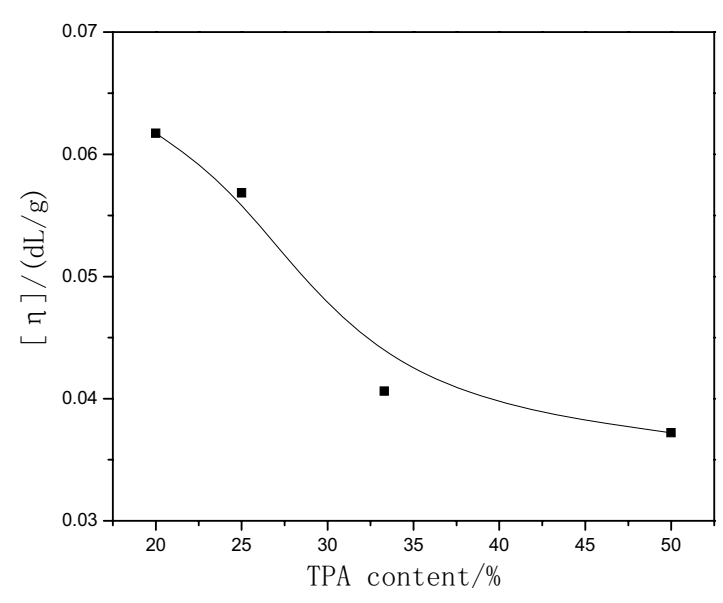

A: Different TPA content

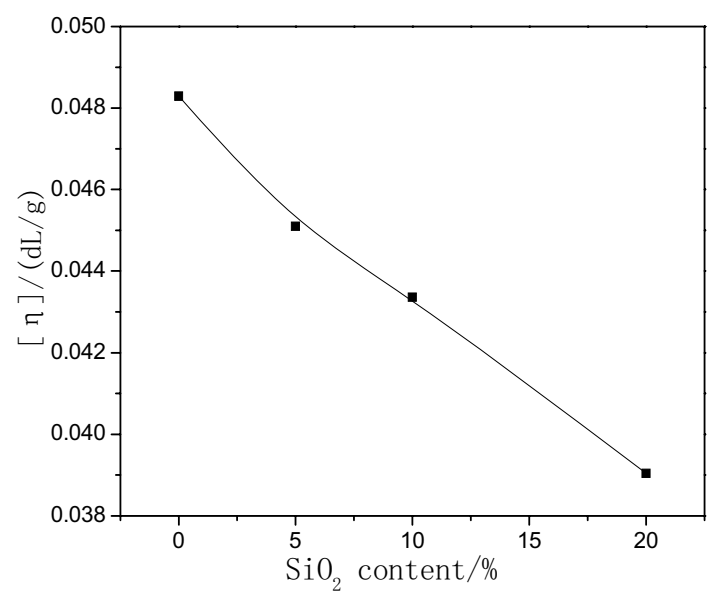

B: Different $\mathrm{SiO}_{2}$ content

Figure 1. The influences of TPA content and $\mathrm{SiO}_{2}$ content on polymer viscosity

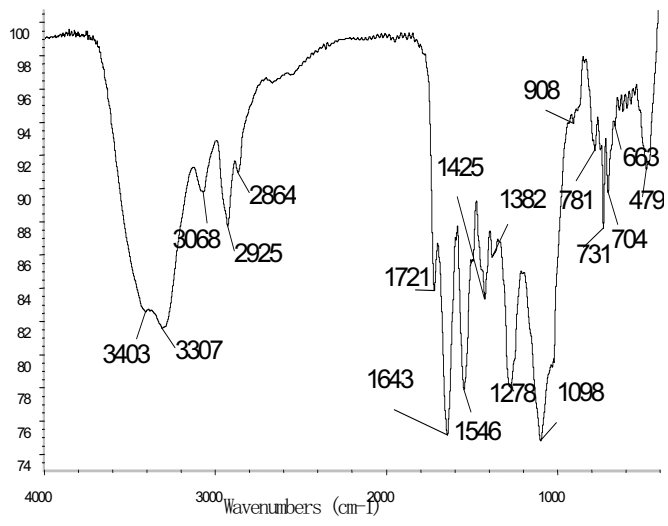

A:MXD6T

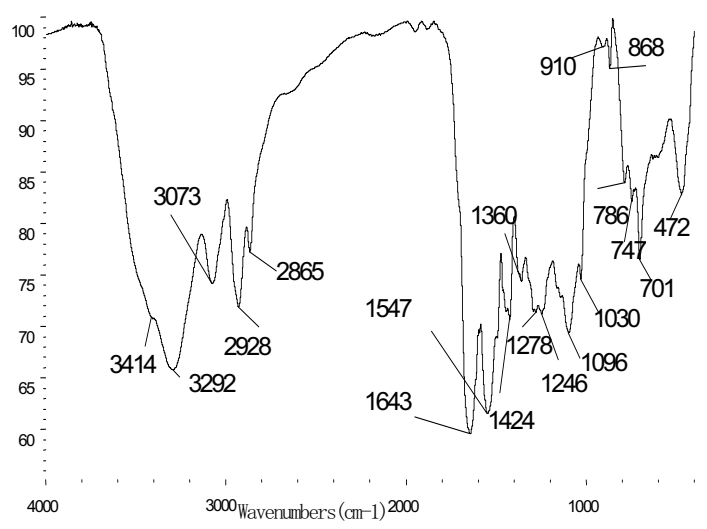

B: NMXD6T

Figure 2. The FITR spectrum of MXD6T and NMXD6T 


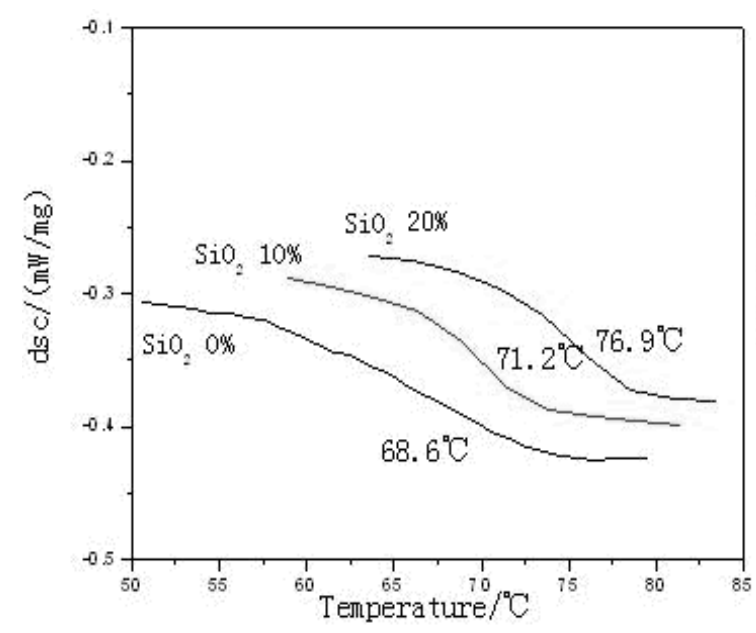

A: different $\mathrm{SiO}_{2}$ concentration of NMXD6T

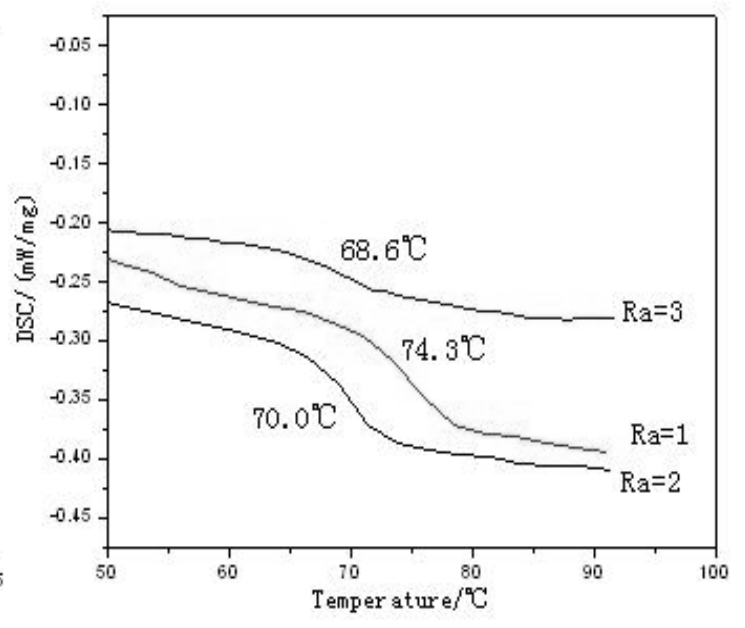

B: different A/TPA value of MXD6T

Figure 3. The glass transition temperatures of oligomers

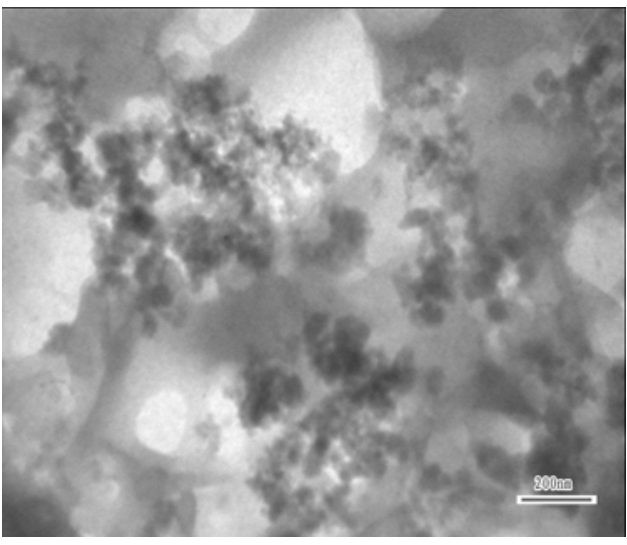

A

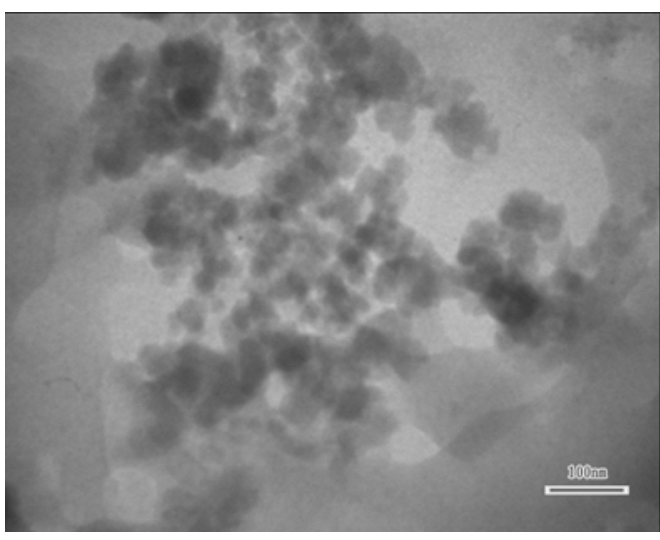

B

Figure 4. The dispersion of nanometer particles in NPET 